\title{
An Elaboration of a Strategic Alignment Model of University Information Systems based on SAM Model
}

\author{
Souad Ahriz \\ SSDIA Laboratory \\ ENSET Mohammedia \\ Hassan II University of Casablanca \\ Mohammedia, Morocco
}

\author{
Nezha Benmoussa \\ SSDIA Laboratory \\ ENSET Mohammedia \\ Hassan II University of Casablanca \\ Mohammedia, Morocco
}

\author{
Abir El Yamami \\ SSDIA Laboratory \\ ENSET Mohammedia \\ Hassan II University of Casablanca \\ Mohammedia, Morocco
}

\author{
Khalifa Mansouri \\ SSDIA Laboratory \\ ENSET Mohammedia \\ Hassan II University of Casablanca \\ Mohammedia, Morocco
}

\author{
Mohammed Qbadou \\ SSDIA Laboratory \\ ENSET Mohammedia \\ Hassan II University of Casablanca \\ Mohammedia, Morocco
}

\begin{abstract}
Information system is a guarantee of the universities' ability to anticipate the essential functions to their development and durability. The alignment of information system, one of the pillars of IT governance, has become a necessity. In this paper, we consider the problem of strategic alignment model implementation in Moroccan universities. Literature revealed that few studies have examined strategic alignment in the public sector, particularly in higher education institutions. Hence we opted for an exploratory approach that aims to better understanding the strategic alignment and to evaluate the degree of its use within Moroccan universities. The data gained primarily through interviews with top managers and IT managers reveal that the alignment is not formalized and that it would be appropriate to implement an alignment model. It is found that the implementation of our proposed model can help managers to maximize returns of IT investment and to increase their efficiency.
\end{abstract}

Keywords-university; business strategy; IT strategy; information system; IS alignment; strategic alignment model

\section{INTRODUCTION}

Higher education institutions offer an increasingly broad range of services. Their information systems are complex because of the variety and volume of information to be processed, the security of information to ensure and of the diversity of users: managers, students, teachers, administrative and external stakeholders. They represent the service production tool and the appropriate support solution in the face of speed, responsiveness, agility and flexibility. The merging of universities, the internationalization and virtualization, the massive number of students, push the information systems to the needs of constant growing. Their governance must be an integral part of the governance of the institution. In effect, a bad governance of the information system would negatively affect the performance of the university, the quality of education and research and the management of internal processes and costs $[1,2]$. The strategic alignment has emerged as a major concern for IT professionals and business leaders [3]. It is an important topic for researchers and practitioners both. Studies have attracted the attention on its importance, [47]. Other research has shown that it provides organizations with the maximum of benefits and a competitive advantage and contributes to the improvement of their organizational performance [8] and to the creation of business value [9].

The majority of these studies concern the industrial and financial fields. Author in [10] found that IT alignment has been limited by the lack of knowledge on the banking sector. In a study on the alignment in the insurance sector, authors in [11] shown that the know-how shared between businesses and IT executives was the strongest predictor of the alignment social dimension. Nevertheless, in the context of education, there is need for more research [12]. Our study proposes to contribute to the understanding of the concept of the alignment through the definitions reported in the literature, to examine the alignment of the strategy of the information system to the business strategy in Moroccan universities and to propose an approach to align these two strategies.

\section{STRATEGIC ALIGNMENT}

Despite the amount of produced research on the strategic alignment, this subject remains as one of the top concerns identified by IT executives and managers. This section provides a portrait of the state of alignment knowledge and presents some alignment models. 


\section{A. Concept of the Strategic Alignment}

Strategic alignment appears in the literature under different terminologies. Among which we find: "fit", "integration" [13], "liaison" [14] "adjustment" [15], "bridge" [16], "harmony" [17], "fusion" [3]. Despite their subtle differences, these terms relate to all the approximations of the strategy of the organization to its information system [18]. The alignment is the dominant term in the literature but other terms are also used such as congruence and covariation. Strategic alignment aims to put in coherence the strategy of an organization and its internal and external functioning, which includes its information system. A number of authors have worked on the strategic alignment, either in a global perspective [19] or focused on more specific aspects of the strategy, such as the information system [18, 20]. Several other definitions of the alignment are proposed in the literature [7, 14, 17, 21]. By browsing through these definitions, we find that there is no consensual definition about the concept of alignment or a detailed description of the elements of the organization involved. But they all agree that there are at least two areas to align, integration of the organizational strategy with the IT strategy and proposed approach support.

\section{B. Alignment Models}

Several models have been proposed to help organizations to achieve IT alignment. Some of them explained the alignment theoretically, others sought to evaluate it. The model of the organizational balance of Scott Morton advocates an alignment between the organizational domains that are the strategy and technology, the roles of individuals and the culture of the organization, the management processes and the structure. Author in [22] stressed four main areas: the business strategy, the IT policy, the organizational strategy and culture, and the financial strategy. Authors in [23] have examined the alignment of IT with the company, while authors in [24] focused on the strategic direction of the business and information systems. Authors in [25] analyzed the relationship between aspects of organizational culture and the IT policy in the units of the public sector. The model in [26] advocates the detailed description of an organizational entity, the application of the architecture principles and the choice of the good level of analysis to make an alignment between the components of this entity with its environment. The model in [17] identifies the enablers and inhibitors of the alignment as well as the criteria for its assessment. The strategic alignment model (SAM) proposed in [13] remains one of the most widely known and used among IS researchers [27]. It is focused on four areas, namely the business strategy, the IT strategy, infrastructure and organizational processes, IT infrastructure and process. The common point between the most of these models is that they incorporate the fact that the strategy often changes and that alignment of IT with the rest of the organization must integrate these strategic changes continuously. Although the models developed in literature are numerous, SAM remains one of the most utilized models in literature and in corporations [18].

\section{The Strategic Alignment Model (SAM)}

The structure of the SAM model contains three classes composed of different elements.
- Domains: Business and Information Technology.

- Levels: Subdivision of areas in strategy for the external level and structure for internal level

- The components: Structuring and characterization of each level in scope, skills and governance for the external level and infrastructure, skills and processes for the internal level.

The concept of the strategic alignment between IT and business is based on two building blocks:

- Strategic fit: It is a link between internal and external domains. It refers to the use of strategy to determine the infrastructure of the business.

- Functional integration between business and IT domain. It takes two distinct forms:

- The strategic integration is the integration between IT strategy and business strategy and establishes the potential of IT at a strategic level.

o The operational integration is the link between organizational infrastructure/ processes and IT infrastructure/processes.

In the external level, the business strategy concerns the decision-making in relation with products and the positioning of the company on the market. The IT strategy concerns the technological scope, the technological skills and IT governance. In the internal level, the organizational structure relates to the process of activities. The technological infrastructure and the processes of IS design concern the technical architecture of IS, the design process, evolution and maintenance of IS as well as the management of technological knowledge and skills. On the basis of these two levels, the model offers four distinct perspectives of alignment that can guide the strategy of an organization in terms of its technological choices: Execution of the strategy, technological transformation, competitive advantage, level of service [27].

\section{RESEARCH MODEL}

The Moroccan university currently knows important organizational changes generated by the legislation in force and by management constraints imposed by its environment (the process of fusion, huge number of students, technological evolution, and infrastructure change). All these variables require universities to restructure their information systems. An IS consists of several heterogeneous applications dedicated to its specific areas in which the mapping is presented in Figure 2. Universities are a special type of organizations where IT alignment has not attracted the researcher attention it deserves $[21,28]$. According to [12] there is a lack of research related to IT aligment in universities. The main concern of their managers is to have an integrated, efficient IS and especially aligned to their overall strategy and to the user expectations. In order to fill this gap and to contribute to the achievement of this strategic objective, our study proposes the adaptation of the mechanisms of SAM and to address a generic approach for modeling the strategic alignment in universities. It consists in characterizing the notion of alignment at the strategic and 
operational level and in maintaining the consistency between all information systems.

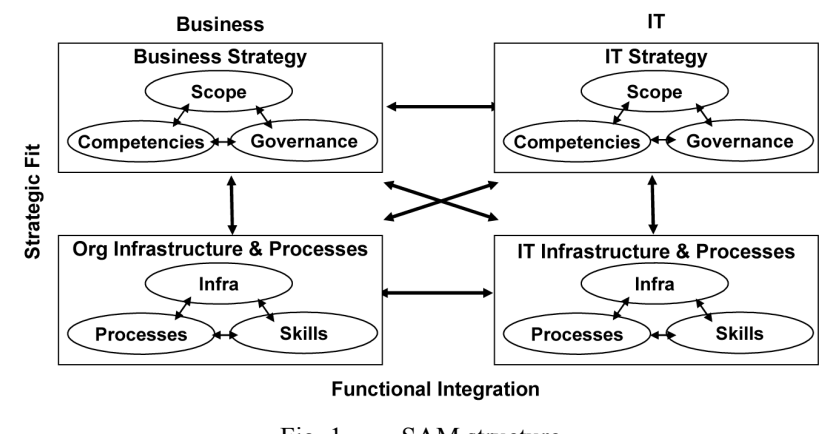

Fig. 1. SAM structure
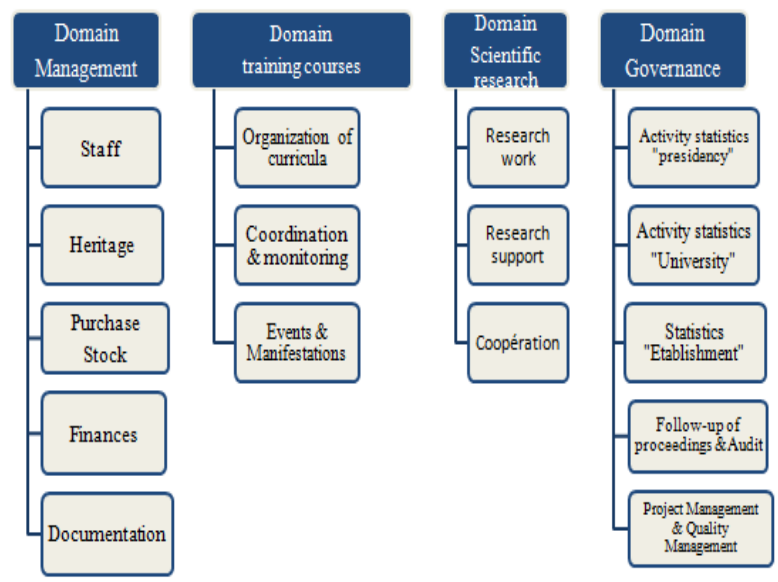

Fig. 2. Mapping of areas of Moroccan universities

The objective of this a model is to show the strategic role that IT plays in enabling organizations to maximize the return on investment in information technology, to provide them a competitive advantage and to increase their efficiency and profitability. The method used in this research is based on an exploratory approach which aims to make an inventory of the apprehension of the concept of alignment by internal information system's users. We have analyzed official documentation relating to Moroccan universities and information gathered through direct interviews with business and IT managers to finally propose the following steps to build our proposed model:

\section{A. Step 1: Selection of the Business Process to Align}

We have chosen to adapt the SAM model to the alignment of the integrated system of accounting and financial management IS FAM, taking into account the strategic role it plays in the university management. This solution allows the management of the whole of the accounting processes and university budget, the preparation and the rapid execution of the annual budget and constitutes a reporting tool and aid to decision-making promoting better strategic thinking. It is used by more than 120 higher education institutions. Nevertheless, some of its modules have been little exploited so far, in spite of their importance due to several reasons among which we quote the resistance to change of certain users, which justifies the proposal for this alignment approach. Understanding the process to align is an essential condition for the achievement of the alignment. As the accounting and budgetary management process was not formalized and in order to facilitate its understanding, we propose hereafter a schematization in terms of operations and management rules which will serve as a basis for harmonizing the methods of identifying needs and possibly standardizing them for all academic institutions.

\section{B. Step 2: Identification and Interpretation of Entities to Align}

The concept of alignment implies the existence of relations between several entities that need to be considered at once. By analogy to the original model, our model baptized SAMU (Strategic Alignment Model for Universities) distinguishes an external level (strategies of the university in terms of finance and accounting, and the strategy of the IS) and an internal level (business processes and management, infrastructure and technological processes) and presents the alignment according to these four dimensions. The different entities of the university can be organized for work harmoniously toward a common goal. We are trying to put in coherence the FAM and IT strategy as well that the FAM process and the IT process to achieve this goal. The implementation of an alignment model requires a well known business strategy to all stakeholders to which the IT strategy can be aligned by bringing its expertise to support business choices. It is for this reason that the communication of the strategy and the information of all the participants in the process of alignment are essential conditions for its success. The following is a description of these entities:

University Strategy: The implementation of the IS FAM aims at a flexible and efficient budget management and thus the improvement of the general framework for steering and strengthening the internal governance system through traceability of various financial transactions and movements, involvement of all departments in the monitoring and sharing of budgetary and financial information. This strategy facilitates audits and management control to ensure transparent accountability and assessment.

IT Strategy: IT strategy makes available a simple, adapted, scalable, secure and modular FAM system. Information systems must therefore be harmonized in order to ensure simple, rapid and coherent interfacing. The IS FAM must be linked to the university reporting platform to provide real-time access to the necessary information. Also, procedures must be put in place to avoid redundancy while ensuring the continuity of the activity, the confidentiality and the automatic data backup as well as the audit and the security of the IS FAM.

Organizational Processes: The formalisation of the budgeting process and communication is crucial. To do this, necessary skills must be developed. Also the sharing of the platform of the FAM system between the institutions and the presidency is mandatory. In addition, it is necessary to ensure the involvement of the staff responsible for economic and financial business in each academic institution for a better use of the system. 


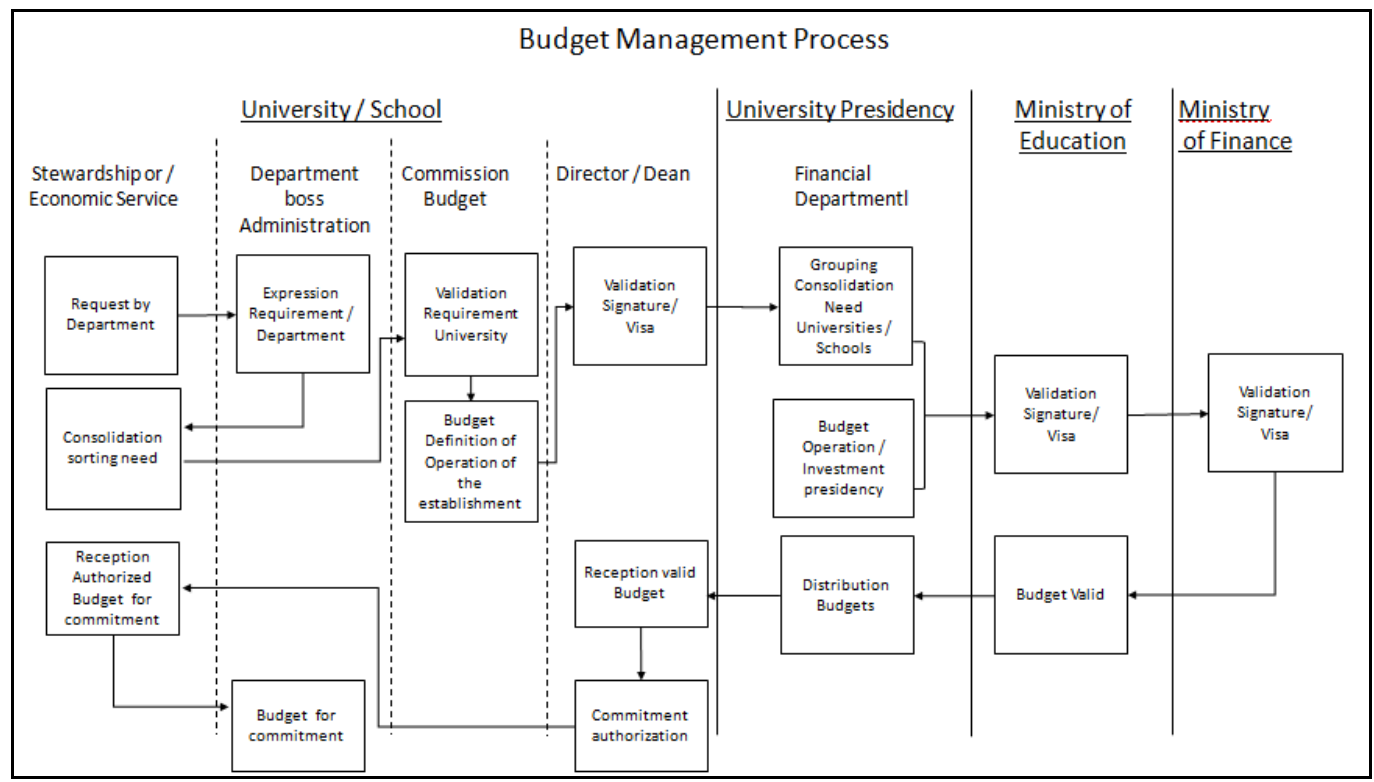

Fig. 3. Needs idendification

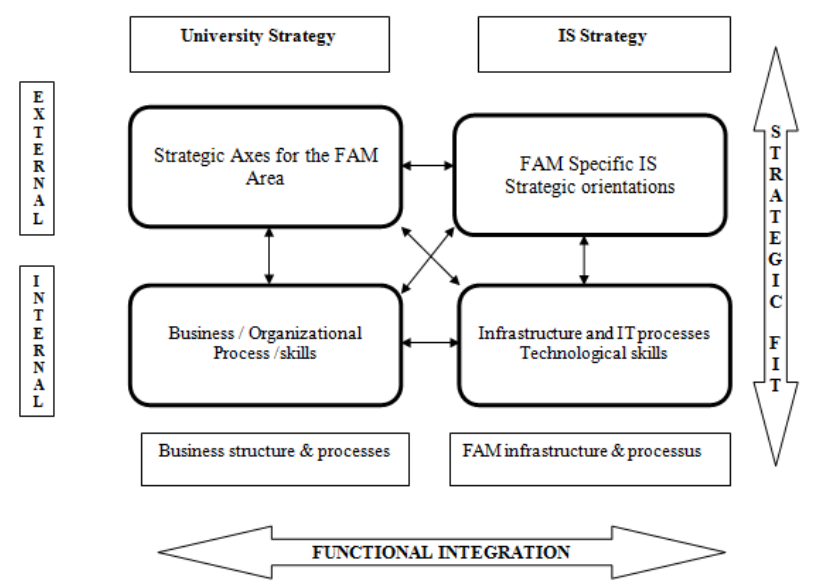

Fig. 4. Structure of the SAMU

IS Process and Infrastructure: For efficiency reasons, a process identity card must be clearly defined. Thus, the choice of systems, applications, databases will be facilitated. The development of a scalable IT infrastructure undoubtedly requires the development of technical and computer skills capable of adapting, analysing business needs, accompanying users in operation and upgrading systems.

\section{Step 3: Identification of Possible Interaction Constraints between These Entities}

An efficient strategic alignment provides a competitive advantage to the organization. To achieve this objective, the overall strategy must be clear and communicated to all stakeholders. The potential of information and communication technologies must be taken into account in the strategy formulation. In addition, a dialogue between business department, top management and IT department must be established to facilitate interactions between entities.

\section{Step 4: Choice of the Alignment Perspective of the SAMU}

Achieving IS FAM alignment with the university's strategy is a first step towards aligning the IS of the university both internally and externally. The alignment approach is to identify the role played by each field in the alignment perspective, establishing relations between different areas and recognize how tasking decisions regarding one of them affects the others. By analogy to the original SAM, each of these perspectives involves three of the four areas to align and involves a strategic fit and a functional integration. To understand the perspectives, we will define three domains:

- The anchor domain is the strongest one in which changes are made. It corresponds to the dimension that the organization controls best. It is the enabler of change that directly impacts the pivot domain.

- The pivot domain is the weakest one where solutions are sought. It implements the anchor which in turn affects the impacted domain.

- The impacted domain corresponds to the domain which will feel the greatest impact from the change in the anchor domain and where the main changes will take place.

Four alignment perspectives emerge from this domain characterization (Figure 5). We have kept the original model outlook because it corresponds well to the case studied. The anchor domain is the strongest domain at the strategic level. The impact domain is located at the internal level at which changes are made.

The university strategy (arrow 1 in Figure 5) is based on business and organizational processes, which in turn must be 
aligned with the IT layer. This first perspective is the most dominant in our case. It is of the type "execution of the business strategy" and is anchored by the choices of the university's strategy (anchor domain). It triggers change needs in the entity "infrastructure and organizational processes" (pivot domain), like developing new skills and justifying the implementation of new processes and technologies (impacted domain). The second sequence (arrow 2) that we propose puts in scene three areas of our SAMU model: business strategy, IT strategy and the IT process. The functional integration between business strategy and IT strategy initiates the sequence of alignment. It is followed by a strategic fit between the IT strategy and IT process. This perspective is to decline the IS FAM strategy through an IT strategy appropriately so that it must be operationalized by identifying the technical infrastructure and the most appropriate IT processes.

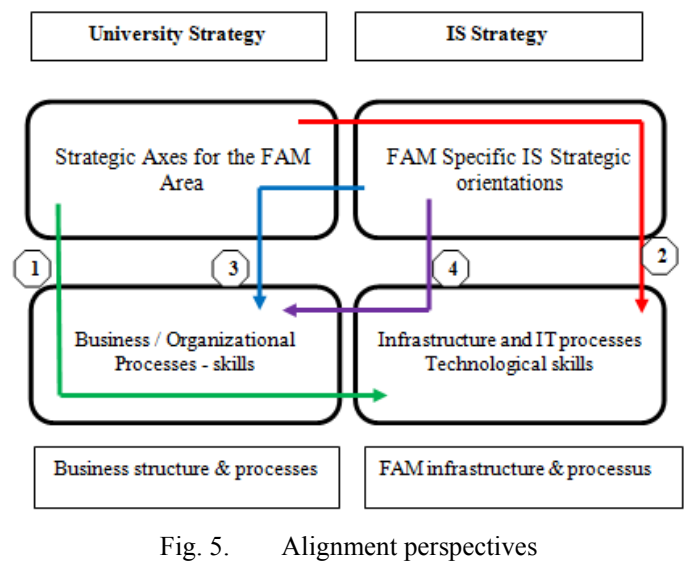

In the third sequence (arrow 3), the technology exploitation is ensured via the IT strategy area of anchor that impacts the organizational process. IT is the driver of change. The business processes are transformed by the adoption of IS FAM. Once the technology is chosen and implemented, the first transformation affects the skills and roles of individuals. These are gradually formed in the use of the new technology. In the fourth perspective (arrow 4), change is initiated by the IT strategy dimension that impacts the entity "organizational processes" thus targeting the IT process in order to provide an excellent level of service to the business process. In general, the choice of the perspective depends on area which requires an evolution and with which there is need to develop the communication and increase understanding. Finally, it is to be noted that the same organization can be brought to follow different modes of alignment depending on the activity and the desired positioning.

\section{RESULTS AND DISCUSSION}

From this study, we can deduct that there is a weak apprehension of the concept of alignment as well as the absence of alignment models use in the Moroccan universities. Literature review revealed that the SAM model is the most adapted or extended in different domains, with the exception of that of higher education, which is the subject of our study. The work presented in this article aimed to introduce a presentation of the strategic alignment and to formalize the alignment approach based on the SAM model in universities. We have therefore formalized the results of the interviews in a model expressing the two strategies (business and IT) and the two processes (business and IT), the main objective is to provide appropriate rules and methods in order to construct the alignment process and to present an approach to facilitate and successfully implement SAM within these institutions.

\section{CONCLUSION AND FUTURE WORK}

In this study, we have used the strategic alignment model (SAM) to compose the structure of SAMU. This model analyzed the relationship between the four areas involved in the alignment process. It will allow university managers to optimally manage IT investments, to optimize resources, to have an integrated and coherent IS able to support the strategy to be implemented and finally to improve the performance of university's processes. In addition to the internal actors of the system, the FAM process also involves external partners such as ministries, suppliers and customers. We plan to work on the full alignment of the system with external partners, the environment and technological developments. We will work on the relationship with the users taking into account the dynamic nature of the alignment due to the increased instability of the environment and its uncertain evolutions.

\section{REFERENCES}

[1] S. Ali, P. Green, "Effective information technology (IT) governance mechanisms: An IT outsourcing perspective", Information Systems Frontiers, Vol. 14, No. 2, pp. 179-193, 2012

[2] M.-S. Pang, G. Lee, W. H. DeLone, "IT resources, organizational capabilities, and value creation in public-sector oragnizations : a publicvalue management perspective", Journal of Information Technology, Vol. 29, No. 3, pp. 187-205, 2014

[3] J. N. Luftman, E. McLean, "Key issues for IT executives", MIS Quarterly Executive, Vol. 3, No. 2, pp. 89-104, 2004

[4] E. McLean, J. V. Soden, Strategic Planning for MIS, 1977

[5] J. C. Henderson, J. G. Sifonis, "The value of strategic IS planning: understanding consistency, validity, and IS markets", MIS Quaterly, Vol. 12, No. 2, pp. 187-200, 1988

[6] R. Almeida, R. Pereira, M. M. da Silva, "IT Governance Mechanisms: A Literature Review", International Conference in Exploring Services Science, pp. 186-199, 2013

[7] W. van Grembergen, S. De Haes, Implementing Information Technology Governance : Models, Practices, and Cases, IGI Publishing, 2007

[8] Y. Chan, S. L. Huff, D. W. Barclay, D. G. Copeland, "Business Strategy Orientation, Information Systems Orientation and Strategic Alignment", Information Systems Research, Vol. 8, No. 2, pp. 125-150, 1997

[9] P. P. Tallon, K. L. Kraemer, V. Gurbaxani, "Executives' Perceptions of the Business Value of Information Technology: A Process-Oriented Approach", Journal of Management Information Systems, Vol. 16, No. 4, pp. 145-173, 2015

[10] W. Baets, “Aligning Information Systems with Business Strategy", The Journal of Strategic Information Systems, Vol. 1, No. 4, pp. 205-213, 1992

[11] B. H. Reich, I. Benbasat, "Factors that influence the social dimension of alignment between business and information technology objectives", MIS Quarterly, Vol. 24, No. 1, pp. 81-113, 2000

[12] K. Walser, D. Weibel, B. Wissmath, S. Enkerli, N. Bigler, M. Topfel, "Business-IT alignment in Municipalities-The Swiss Case", 22nd Americas Conference on Information Systems, pp. 1-10, 2016 
[13] J. C. Henderson, H. Venkatraman, "Strategic alignment: Leveraging information technology for transforming organizations", IBM Systems Journal, Vol. 38, No. 2, pp. 472-484, 1993

[14] B. H. Reich, I. Benbasat, "Measuring the Linkage between Business and Information Technology Objectives", MIS Quarterly, Vol. 20, No 1, pp. 55- 81, 1996

[15] M. E. Porter, "What is Strategy ?", Harvard Business Review, Nov.-Dec. ,pp. 61-78, 1996

[16] C. U. Ciborra, "De profundis? Deconstructing the concept of strategic alignment", Scandinavian Journal of Information Systems, Vol. 9, No. 1, pp. 67-82, 1997

[17] J. N. Luftman, T. Brier, "Achieving and sustaining business-IT alignment", California Management Review, Vol. 42, No 1, pp. 109122, 1999

[18] D. Avison, J. Jones, P. Powell, D. Wilson, "Using and validating the strategic alignment model" Journal of Strategic Information Systems, Vol. 13, No. 3, pp. 223-246, 2004

[19] R. S. Kaplan, D. P. Norton, L'alignement stratégique, Créer des synergies par le tableau de bord prospectif, Editions d'Organisation 1ère édition, 2007

[20] M. Kalika, Y. Rival, "Measuring Internet performance within the organization", 16th Information Resources Management Association International Conference, USA, 2005

[21] T. J. Winkler, "IT governance mechanisms and administration/IT alignment in the public sector: A conceptual model and case validation", Proceedings of International Conference on Wirtschaftsinformatik, pp. $831-845,2013$

[22] H. Pankratz, "Strategic Alignment: Managing for Synergy", Business Quarterly, Vol. 55, No. 3, pp. 66-67, 1991

[23] C. V. Brown, S. L. Magill, "Alignment of the IS function with the enterprise: towards a model of antecedents" MIS Quarterly, Vol. 14, No. 8, pp 371-403, 1994

[24] Y. E. Chan, B. H. Reich, "IT alignment: what have we learned?", Journal of Information Technology, Vol. 22, No 4, pp. 297-315, 2007

[25] S. Kanungo, S. Sadavarti, Y. Srinivas, "Relating IT strategy and organizational culture: an empirical study of public sector units in India", Journal of Strategic Information Systems, Vol. 10, No. 1, pp. 2957,2001

[26] J. A. Zachman, "A framework for information systems architecture", IBM Systems Journal, Vol. 26, No. 3, pp. 276-292, 1987

[27] A. Renaud, I. Walsh, M. Kalika, "Is SAM still alive? A bibliometric and interpretive mapping of the strategic alignment research field", The Journal of Strategic Information Systems, Vol. 25, No. 2, pp. 75-103, 2016

[28] S. Vander Elst, F. De Rynck, "Alignment processes in public organizations: an interpretive approach", Information Polity, Vol. 19, No 3,4 , pp. $195-206,2014$ 